\title{
Time-trend and recurrence analysis of pancreatic neuroendocrine tumors
}

\author{
Hanbaro Kim¹, Ki Byung Song², Dae Wook Hwang², Jae Hoon Lee², Shadi Alshammary³ and Song Cheol Kim² \\ 1'Department of Surgery, Hallym University College of Medicine, Chuncheon, Republic of Korea \\ 2Division of Hepatobilliary and Pancreatic Surgery, Department of Surgery, University of Ulsan College of Medicine and Asan Medical Center, Seoul, \\ Republic of Korea \\ ${ }^{3}$ Department of Surgery, College of Medicine, Imam Abdulrahman Bin Faisal University, Dammam, Saudi Arabia
}

Correspondence should be addressed to S C Kim: drksc@amc.seoul.kr

\begin{abstract}
This study aimed to evaluate the evolving trends in clinicopathological features of pancreatic neuroendocrine tumors and to analyze the predictors of recurrence after curative resection. Data collected retrospectively from a single center between January 1990 and December 2017 were analyzed. Patients were categorized chronologically into three groups for evolving time-trend analysis. Overall, 542 patients (300 female, 55.4\%) underwent surgical resection for pancreatic neuroendocrine tumors, including 435 (80.3\%) with non-functional tumors. Time-trend analysis revealed that the surgically resected pancreatic neuroendocrine tumor number increased consistently; however, the incidental non-functional pancreatic neuroendocrine tumor number also increased over time $(P<0.001)$. The 5 - and 10-year disease-free survival rates were 86.4 and $81.3 \%$, respectively. The overall recurrence rate was $13.7 \%$, and the most common site of recurrence was the liver. The median time to recurrence after primary surgery was 19.0 (range 0.8-236.3) months, and the median survival time after recurrence was 22.6 (range 0.4-126.9) months. On multivariate analysis, grade G3 pancreatic neuroendocrine tumors (hazard ratio 4.51; $P<0.001$ ), lymph node metastasis (hazard ratio 2.46; $P=0.009$ ), lymphovascular invasion (hazard ratio $3.62 ; P=0.004$ ), perineural invasion (hazard ratio $2.61 ; P=0.004$ ) and resection margin (hazard ratio 4.20; $P=0.003$ ) were independent prognostic factors of disease-free survival. The surgically resected pancreatic neuroendocrine tumor number increased over time mainly because of an increase in incidentally discovered non-functional pancreatic neuroendocrine tumors. Grade G3 pancreatic neuroendocrine tumors, lymph node metastasis, lymphovascular invasion, perineural invasion and a positive resection margin were significant predictors of worse disease-free survival in patients with surgically resected pancreatic neuroendocrine tumors.
\end{abstract}
Key Words
- pancreatic NET
- recurrence
- time-trend
- predictor

\section{Introduction}

Pancreatic neuroendocrine tumors (PNETs), also known as islet cell tumors, are rare neoplasms arising from the endocrine pancreas, with a reported incidence of $<1$ per 100,000 persons per year $(1,2)$. However, the incidence of PNETs has been steadily increasing, possibly as a result of increased detection on endoscopic and cross-sectional imaging studies $(3,4)$. PNETs are functional or non-functional tumors and represent a heterogeneous group of neoplasms with extremely variable clinical behavior. Functional PNETs (F-PNETs) are diagnosed on the basis of the presence of clinical symptoms such as hypoglycemia, diarrhea or peptic ulcer. Non-functional PNETs (NF-PNETs) are often discovered later, that is, at an advanced stage, because there are no specific clinical
Endocrine Connections (2019) 8, 1052-1060 
symptoms related to hormone excess. However, in recent years, non-functional tumors have been discovered incidentally in patients undergoing diagnostic evaluation for unrelated conditions $(4,5)$. Therefore, the outcome of symptomatic presentation versus incidental diagnosis in patients with a NF-PNET has received increasing interest. Some studies have reported that the incidental diagnosis of PNETs was associated with improved survival following resection with curative intent $(6,7)$.

Currently, complete surgical removal is considered the first-line treatment for patients with PNET, although the recommendation for surgery is size dependent for NF-PNETs. The primary goal of surgical resection is curative, while secondary goals are symptom control and limitation of tumor progression. Although PNETs are reported to display an indolent course and are associated with longer survival when compared with other pancreatic neoplasms such as ductal adenocarcinoma, some PNETs recur. The natural history of PNETs is not fully understood because of their rarity; therefore, it is difficult to precisely predict the malignant potential of PNETs. Moreover, in the 2010 World Health Organization (WHO) classification, poorly differentiated neuroendocrine carcinomas are recurrent and have a poor prognosis. However, there have been only a few large-sample studies investigating the pattern and prognostic factors of recurrence of PNETs.

The aim of this study was to assess the evolving trends in the clinicopathological features of PNETs by dividing a single-center patient series conducted over a 27-year period into three groups. Furthermore, this study evaluated the pattern and predictors of recurrence after curative resection of such tumors.

\section{Materials and methods}

\section{Data collection}

The medical records of patients with PNET who underwent surgical treatment at the Asan Medical Center/Ulsan
University School of Medicine, Korea between November 1990 and December 2017 were retrospectively reviewed. A total of 542 patients were included in this study for timetrend analysis (Fig. 1), and they were categorized into three groups according to treatment date (1990-2000, $n=34 ; 2001-2010, n=199$ and 2011-2017, $n=309$ ).

Data related to mortality, age, sex, type of operation, sites of recurrence and type of treatment were collected and reviewed. Patients with signs, symptoms and biochemical evidence of hormonal excess were considered to have a functional tumor. Patients with no recognizable clinical syndrome and normal serum hormone levels were considered to have a non-functional tumor. Patients were classified as 'symptomatic' when clinical symptoms such as weight loss, abdominal pain, hypoglycemia or diarrhea were present at diagnosis. Asymptomatic PNETs were defined as tumors incidentally discovered in asymptomatic patients who underwent abdominal imaging for an unrelated cause. Data on clinical presentation, demographics and surgical procedures, such as postoperative course and complications, pathology and follow-up, also were collected. The Institutional Review Board of Asan Medical Center certified this study (S2018-2397-0001).

\section{Preoperative work-up, surgical procedure and pathological analysis}

Preoperative radiological assessment included abdominal and pelvic computed tomography (CT) and, in selected cases, magnetic resonance imaging (MRI) of the pancreas, as well as the liver, if metastases or suspicious liver lesions were detected on CT. Surgical resection with curative intent involved the complete resection of all identified disease at operation, including the primary tumor, hepatic metastases and other intra-abdominal diseases, as necessary. Surgical procedures included pancreaticoduodenectomy (PD), distal pancreatectomy (DP), total pancreatectomy (TP), central pancreatectomy (CP) and enucleation (EN). Ablation was performed for

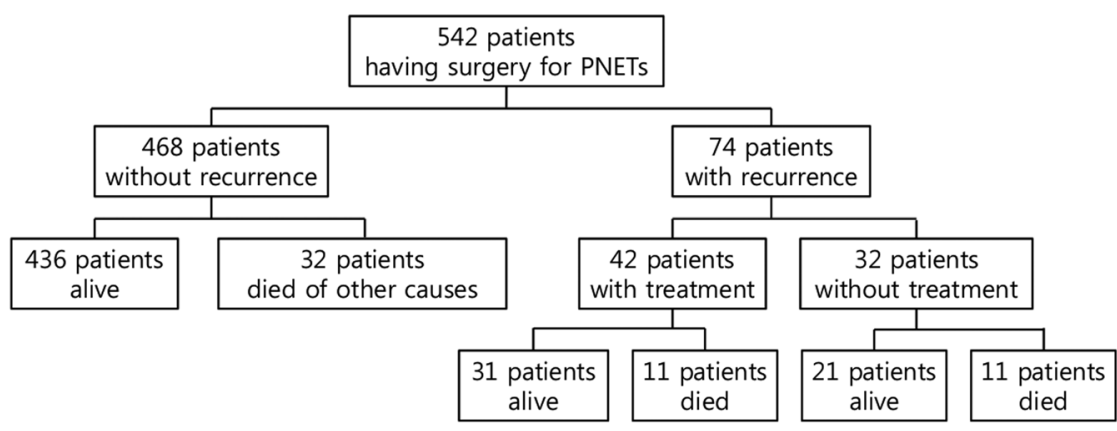

Figure 1

Study flow diagram. We conducted a retrospective review of 542 patients with surgically resected PNETs. Of these patients, 74 patients underwent recurrence. https://ec.bioscientifica.com https://doi.org/10.1530/EC-19-0282
(C) 2019 The authors Published by Bioscientifica Ltd

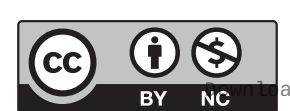

This work is licensed under a Creative Commons Attribution-NonCommercial 4.0 International License. ded from Bioscientifica.com at 04/26/2023 09:11:53AM via free access 
patients with synchronous liver metastasis, hepatectomy or radiofrequency. All patients in this cohort with portal vein invasion also underwent portal vein resection. Our center has the following polices for surgical resection of PNETs: (1) F-PNETs are surgically resected; (2) for NF-PNETs in the body and tail, tumors less than $1 \mathrm{~cm}$ are observed and tumors greater than $1 \mathrm{~cm}$ are surgically resected; (3) for NF-PNETs in the head, tumors less than $2 \mathrm{~cm}$ are observed and tumors greater than $2 \mathrm{~cm}$ are surgically resected if comorbidities are not very high; (4) hepatic metastatic PNETs are resected if they are resectable. These policies have not changed and laparoscopic surgery has been increasing since minimally invasive surgery was introduced in 2006. For morbidity analysis, complications were classified according to the ClavienDindo classification system. Pancreatic fistula was defined and graded according to the International Study Group on Pancreatic Fistula (ISGPF) (8). We also examined mortality, which was defined as death within 90 days of the operation or before discharge from the hospital. The pathologic diagnosis of PNET was based on classic histological and immunohistochemical features. The surgical specimens of all cases were classified according to the 2010 WHO classification criteria. All PNETs were classified according to a grading scheme based on the mitotic count or Ki67 index, as follows: G1 (mitotic count $<2 / 10$ high-power fields (HPFs) and/or $\leq 2 \%$ Ki67 index), G2 (mitotic count 2-20/10 HPF and/or 3-20\% Ki67 index) and G3 (mitotic count $>20 / 10 \mathrm{HPF}$ and/or $>20 \% \mathrm{Ki} 67$ index). The 7th edition of the American Joint Committee on Cancer (AJCC) recommends that the tumor-nodemetastasis (TNM) staging system also be used for tumor staging: T1, tumor limited to the pancreas and size $<2 \mathrm{~cm}$; $\mathrm{T} 2$, tumor limited to the pancreas and size greater than $2 \mathrm{~cm}$; T3, tumor extending beyond the pancreas and T4, tumor invading the celiac axis or superior mesenteric artery (SMA). Primary tumor lymphovascular invasion (LVi), perineural invasion (PNi) and resection margins were also evaluated in pathological samples.

Somatostatin analogs were not routinely used after surgery. Adjuvant chemotherapy (including etoposide and cisplatin (EP) and capecitabine) and target therapy (including everolimus and sunitinib) were administered when recommended by oncologists.

\section{Follow-up}

Survival status was determined from national registries. Death certificates and time of death were confirmed by a National Health Insurance inquiry. All patients were followed up periodically according to tumor grade to monitor tumor recurrence. Patients with G1 grade PNETs were followed up at 3 months after surgery and annually thereafter. For patients with G2 grade PNETs, we followed up at 3 months after surgery, every 6 months for 2 years and then annually thereafter. Finally, follow-up was every 3 months for 2 years, and then every 6 months for 5 years, and then every 1 year thereafter for patients with G3 grade PNETs. The end of follow-up was July 2018. Overall survival (OS) was defined as the interval between the date of surgery and the date of death, and disease-specific survival (DSS) was defined as the interval between the date of primary resection and the date of death from disease progression. Disease-free survival (DFS) was defined as the duration between primary resection and the first documented progression of recurrent disease on CT or positron-emission tomography (PET) during regular follow-up. Tumor recurrence was defined as progressive soft tissue growth and hypermetabolic lesions at specific sites. Histopathological confirmation was not routinely performed for the diagnosis of tumor recurrence. After recurrence, multi-disciplinary treatment, such as hepatectomy, transarterial chemoembolization (TACE), and radiofrequency ablation (RFA) for hepatic recurrence, along with somatostatin analog administration, chemotherapy and target therapy, was performed. Survival time after recurrence (SAR) was defined as the interval between the first documented recurrence and death. The SAR of patients with at least one treatment or multi-disciplinary treatment was compared with that of patients without treatment.

\section{Statistical analysis}

Continuous variables are expressed as medians and ranges or mean \pm s.D.). Categorical variables are reported as numbers and percentages. ANOVA was used for timetrend analysis of the mean tumor size. The OS, DSS and DFS from the date of surgery were calculated using the Kaplan-Meier method. The SAR also was calculated using the Kaplan-Meier method. The log-rank test was used for group comparisons. Statistical analyses were performed using SPSS software (version 22.0; SPSS Inc.). Cox's regression analysis was performed to identify factors independently associated with prognosis. Multivariate analysis included clinically important parameters identified on univariate analysis and those with $P<0.050$. Hazard ratios (HRs) and 95\% confidence intervals (CIs) are presented for all variables.

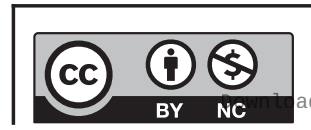

This work is licensed under a Creative Commons Attribution-NonCommercial 4.0 International License. ded from Bioscientifica.com at 04/26/2023 09:11:53AM 


\section{Results}

\section{Study population}

From January 1990 to December 2017, a total of 542 patients (300 female, 55.4\%) underwent curative surgical resection for PNET. The median age at diagnosis was 53 (range 43-62) years. Initial presentation was accompanied by symptoms such as abdominal pain, weight loss, hypoglycemia and diarrhea in 272 (50.2\%) patients. There were 258 (47.6\%) asymptomatic patients. A total of 435 (80.3\%) patients had NF-PNET. The median tumor size was 20.0 (range 14.0-37.3) $\mathrm{mm}$.

According to the 2010WHOclassification, 250(46.1\%) patients were diagnosed with a G1 tumor, 154 (28.4\%) patients with a G2 tumor and 33 (6.3\%) patients with a G3 tumor. According to the 7th AJCC-recommended TNM staging system, 429 (79.9\%) patients had a T1/T2 tumor and 113 (20.8\%) had a T3/T4 tumor. In total, $67(12.4 \%)$ patients had lymph node metastasis and 20 (3.7\%) patients had synchronous metastasis involving the liver (19 patients, 3.5\%) and stomach (1 patient, $0.2 \%$ ). Whereas $\mathrm{PNi}$ was identified in 82 (15.1\%) patients, LVi was identified in 122 (22.5\%) patients. Thirty-one (5.7\%) patients had a positive resection margin.

A total of 249 (45.9\%) patients underwent DP, 199 (36.7\%) underwent PD, 50 (9.2\%) underwent EN and $32(5.9 \%)$ underwent CP. Twelve (2.2\%) patients underwent TP. Sixteen (3.0\%) patients required portal vein resection due to portal vein involvement. Only 28 (5.2\%) patients subsequently underwent adjuvant therapy. One patient $(0.2 \%)$ with a T4 tumor underwent SMA resection and anastomosis. Among the 19 patients with synchronous liver metastasis, 16 underwent hepatectomy and 7 underwent intraoperative radiofrequency ablation (alone or together with hepatectomy). One patient with synchronous stomach metastasis underwent stomach wedge resection.

After surgery, $11(2.0 \%)$ patients received adjuvant chemotherapy, 10 (1.8\%) received somatostatin analogs, $4(0.8 \%)$ received targeted therapeutic agents like everolimus and sunitinib and $2(0.4 \%)$ received radiotherapy.

The overall morbidity rate was $22.5 \%$. Thirtyeight (7.0\%) patients developed a clinically relevant postoperative pancreatic fistula (ISGPF grade B/C). One patient $(0.2 \%)$ died in hospital due to a non-surgeryrelated complication (i.e., stroke). The demographics and surgical and pathological outcomes are summarized in Table 1.

\section{Time-trend analysis}

During the study period, the overall number of cases of PNETs constantly increased, with more than 40 cases per year compared to the previous 6 years (Fig. 2). The proportion of asymptomatic patients with incidentally discovered PNET also increased, from 3\% between 1990 and 2000 (the 'earliest period') to 40\% between 2001 to 2010 (the 'early period'), and finally to $59.3 \%$ between 2011 and 2017 (the 'late period') $(P<0.001)$; the rate of NF-PNET also increased, from 38.2 to 71.9 to $90.3 \%$, respectively $(P<0.001$; Fig. 3$)$. A variation in the total mean tumor size was observed from a mean of $24.8 \mathrm{~mm}$ in 2000 to a mean of $29.1 \mathrm{~mm}$ in the early period, and finally up to a mean of $28.1 \mathrm{~mm}$ in the late period $(P=0.130)$ (Table 2). However, the mean tumor size of NF-PNETs decreased significantly from $39.2 \mathrm{~mm}$ to $32.2 \mathrm{~mm}$ to $29.0 \mathrm{~mm}$ (earliest, early and late periods, respectively) $(P=0.045$; Table 2). With respect to pathology, tumor grade analysis showed that the prevalence of G1 tumor decreased from $80.8 \%$ in the earliest period to $69.8 \%$ in the early period, and finally to $50.5 \%$ in the late period. The rate of G2 tumor increased from 13.3\% in the earliest period to $19.0 \%$ in the early period, and finally to $43.1 \%$ in the late period. The rate of G3 tumor in the late period was lowest, at $6.4 \%$, compared to $11.1 \%$ in the early period and $6.7 \%$ in the earliest period. When DSS and DFS of NF-PNETs were analyzed by period, a trend toward improved DSS and DFS was identified, albeit this was not statistically significant $(P=0.068$ and $P=0.627$, respectively; Table 3).

\section{Recurrence patterns}

During the follow-up period, 74 (13.7\%) patients experienced recurrence, with a median time to recurrence after surgery of 19.0 (range 0.8-236.3) months. The most common organ associated with recurrence was the liver. Of 62 patients with liver metastasis, 5 also experienced simultaneous recurrence in the peritoneum, 1 in the remaining pancreas, 1 in the lung and supraclavicular lymph node and 1 in bone. Of the 12 patients who experienced recurrence in an organ other than the liver, 4 had recurrence in the retroperitoneum, 3 in the peritoneum, 2 in the stomach, 1 in the skin, 1 in bone and 1 in the lung (Table 4). In total, 61 patients developed recurrence within 5 years and 10 developed recurrence between 5 and 10 years, with 3 patients dying due to related causes. Three patients also developed hepatic recurrence after 10 years but survived.

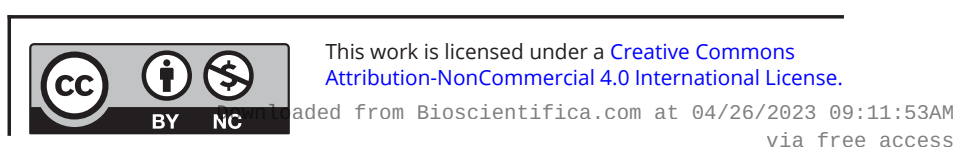


Table 1 Demographic, surgical and pathological data of the study population.

\begin{tabular}{|c|c|}
\hline & $\begin{array}{l}\text { All patients } \\
(n=542)\end{array}$ \\
\hline Sex - female & $300(55.4)$ \\
\hline Age (year) ${ }^{a}$ & $53(43-62)$ \\
\hline Symptomatic & $272(50.2)$ \\
\hline Incidental & $258(47.6)$ \\
\hline Non-functional tumor & $435(80.3)$ \\
\hline Functional tumor & $107(19.7)$ \\
\hline Insulinoma & $103(18.9)$ \\
\hline Glucagonoma & $1(0.2)$ \\
\hline Somatostatinoma & $1(0.2)$ \\
\hline VIPoma & $2(0.4)$ \\
\hline \multicolumn{2}{|l|}{ Tumor location } \\
\hline Head/neck/uncinate & $256(47.2)$ \\
\hline Body/tail & $286(52.8)$ \\
\hline Multiple & $23(4.2)$ \\
\hline Tumor size $(\mathrm{mm})^{\mathrm{b}}$ & $22.0(14.0-37.3)$ \\
\hline Synchronous liver metastasis & $19(3.5)$ \\
\hline \multicolumn{2}{|l|}{ Surgical approach } \\
\hline Open & $273(50.4)$ \\
\hline Minimally invasive & $269(49.6)$ \\
\hline \multicolumn{2}{|l|}{ Type of resection } \\
\hline PPPD or PD & $199(36.7)$ \\
\hline Distal pancreatectomy & $249(45.9)$ \\
\hline Central pancreatectomy & $32(5.9)$ \\
\hline Enucleation & $50(9.2)$ \\
\hline Total pancreatectomy & $12(2.2)$ \\
\hline \multicolumn{2}{|l|}{ Combined operation } \\
\hline Hepatectomy and/or intraoperative RFA & $19(3.5)$ \\
\hline Portal vein resection & $16(3.0)$ \\
\hline Others & $1(0.2)$ \\
\hline \multicolumn{2}{|l|}{ Morbidity (Dindo and Clavien's classification) } \\
\hline Overall & $122(22.5)$ \\
\hline Grade I/II & $94(17.3)$ \\
\hline Grade III/IV & $27(5.0)$ \\
\hline \multicolumn{2}{|l|}{ Pancreatic fistula } \\
\hline Grade A & $102(18.8)$ \\
\hline Grade B & $34(6.3)$ \\
\hline Grade C & $4(0.7)$ \\
\hline Postoperative mortality & $1(0.2)$ \\
\hline Adjuvant treatment & $27(5.0)$ \\
\hline \multicolumn{2}{|l|}{ Grade } \\
\hline G1 & $250(46.1)$ \\
\hline $\mathrm{G} 2$ & $154(28.4)$ \\
\hline G3 & $34(6.3)$ \\
\hline Unknown & $104(19.2)$ \\
\hline \multicolumn{2}{|l|}{ AJCC tumor category } \\
\hline $\mathrm{T} 1 / \mathrm{T} 2$ & $429(79.2)$ \\
\hline T3/T4 & $113(20.8)$ \\
\hline N1 status & $67(12.4)$ \\
\hline M1 status & $20(3.7)$ \\
\hline Lymphovascular invasion & $122(22.5)$ \\
\hline Perineueral invasion & $82(15.1)$ \\
\hline Resection margin & $31(5.7)$ \\
\hline
\end{tabular}

Values in parentheses are percentages.

${ }^{a}$ Values are median (i.q.r.). ${ }^{b}$ Values are median (i.q.r.).

AJCC, American Joint Committee on Cancer; G, tumor grade (World Health Organization classification, 2010); PD, pancreaticoduodenectomy; PPPD, pylorus-preserving pancreaticoduodenectomy; RFA, radiofrequency ablation.

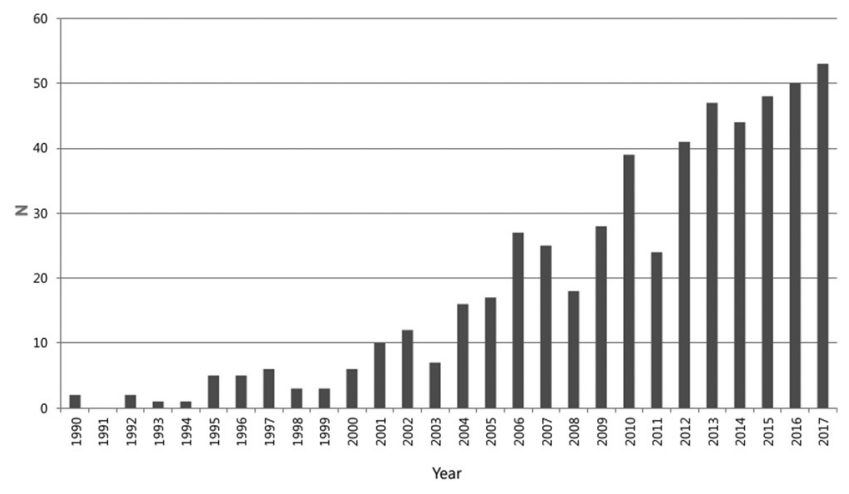

Figure 2

Cases with surgically resected pancreatic neuroendocrine tumors (PNETs) at a single center between 1990 and 2017.

\section{Long-term outcomes}

The median surveillance period was 60.1 (25.7-111.0) months. Of 64 patients who died, 32 (5.9\%) died of other causes, and the remaining $478(88.1 \%)$ were still alive at the end of the surveillance period. The estimated median DSS time was 60.1 (26.1-110.8) months and the resulting 5- and 10-year DSS rates were 94.1 and $90.4 \%$, respectively. The estimated median DFS time was 54.0 (23.1-107.6) months and the 5- and 10-year DFS rates were 86.4 and $81.3 \%$, respectively (Fig. 4). On univariate analysis, factors significantly associated with DFS included nonfunctional tumors $(P<0.001)$, G3 grade $(P<0.001)$, T3/T4 $(P<0.001), \mathrm{N} 1$ status $(P<0.001), \mathrm{M} 1$ status $(P<0.001)$, LVi $(P<0.001)$, PNi $(P<0.001)$ and R1 status $(P<0.001)$. Variables associated with DFS on univariate analysis are shown in Table 4. On multivariate analysis, G3 (HR 4.51; 95\% CI, 2.32-8.78; $P<0.001$ ), N1 status (HR 2.46; 95\% CI, 1.25-4.83; $P=0.009$ ), LVi (HR 3.62; 95\% CI, 1.57-8.34;

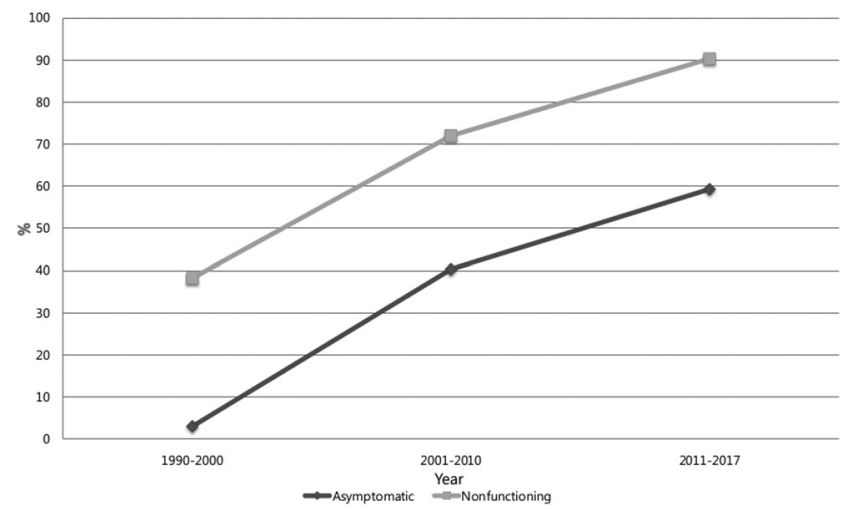

Figure 3

Evolving trend in clinicopathological features of surgically resected pancreatic neuroendocrine tumors (PNETs) during the study period. $P<0.001$ (asymptomatic), $P<0.001$ (non-functioning) $\left(\chi^{2}\right.$ test).

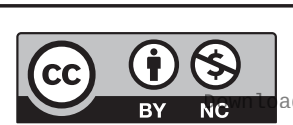

This work is licensed under a Creative Commons Attribution-NonCommercial 4.0 International License. ded from Bioscientifica.com at 04/26/2023 09:11:53AM 
Table 2 Time-trend analysis of the mean tumor size during the study period.

\begin{tabular}{|c|c|c|c|c|}
\hline & 1990-2000 & 2001-2010 & 2011-2017 & $\mathbf{P}$ \\
\hline Total size (mm) & 24.8 & 29.1 & 28.1 & 0.130 \\
\hline NF-PNETs (mm) & 39.2 & 32.3 & 29.0 & 0.045 \\
\hline F-PNETs (mm) & 16.0 & 20.7 & 19.5 & 0.128 \\
\hline
\end{tabular}

F-PNETs, functioning pancreatic neuroendocrine tumors; NF-PNETs, non-functioning pancreatic neuroendocrine tumors.

$P=0.002)$, PNi (HR 2.61; 95\% CI, 1.36-5.01; $P=0.004)$ and R1 status (HR, 4.20; 95\% CI, 1.58-11.12; $P=0.004)$ were significantly associated with decreased DFS in patients with resected PNETs (Table 5).

\section{Post-recurrence survival}

The median SAR was 22.6 (range 0.4-126.9) months. Among the 74 patients who experienced recurrence, 32 (5.9\%) deaths were directly attributed to disease progression. The remaining 42 patients with recurrence were still alive at the time of follow-up. When analyzing survival after recurrence, there was no significant difference $(P=0.06)$ in SAR between the treated $(n=52)$ and untreated $(n=22)$ groups (Fig. 5).

\section{Discussion}

This is the largest single-center retrospective study focusing on the evolving trend and recurrence of PNETs in South Korea. The incidence of PNET has increased over the past few decades because of the more frequent use of endoscopic and cross-sectional imaging techniques and due to increased awareness among physicians $(9,10)$. Specifically, there has been an increase in the reported incidence of incidentally discovered NF-PNETs (11). Incidental NF-PNETs are associated with lower tumor stages, smaller tumor size and a higher number of curative resections (7). Another study also reported a strong correlation between tumor size and malignancy in NF-PNETs (12). In addition, some studies reported that incidental diagnosis was associated with improved

Table 3 Five-year disease-specific survival and 5-year disease-free survival of NF-PNETs according to time period.

\begin{tabular}{|c|c|c|c|c|}
\hline & 1990-2000 & 2001-2010 & 2011-2017 & $P$ \\
\hline 5-year DSS (\%) & 81.8 & 91.3 & 94.7 & 0.068 \\
\hline 5-year DFS (\%) & 76.9 & 81.2 & 84.4 & 0.627 \\
\hline
\end{tabular}

DFS, disease-free survival; DSS, disease-specific survival.
Table 4 Initial PNET recurrence sites.

\begin{tabular}{l}
\hline Recurrent site \\
\hline Liver only \\
Liver + peritoneum \\
Liver + pancreas \\
Liver + lung + supraclavicular lymph node \\
Liver + bone \\
Retroperitoneum \\
Peritoneum \\
Stomach \\
Skin \\
Bone \\
Lung
\end{tabular}

\begin{tabular}{c}
\hline No. of patients $(n=74)$ \\
\hline 54 \\
5 \\
1 \\
1 \\
1 \\
4 \\
3 \\
2 \\
1 \\
1 \\
1
\end{tabular}

survival after curative pancreatic resection, likely because these tumors were diagnosed at an earlier stage $(6,7)$. Our study also showed a trend toward an increase in proportion and number of incidentally discovered NF-PNETs, with an increasing total number of cases of PNETs over time. Moreover, the total mean tumor size was smaller in the late period than in the early period, with a significantly decreased mean tumor size of NF-PNETs over time. When we analyzed the tumor grade over time, the proportion of grade G3 tumors was lowest (6.6\%) in the late period. In the survival analysis, we found that DSS and DFS according to each time period tended to improve, although nonsignificantly. These improvements in survival outcomes may be associated with lower rates of malignancy, as well as a smaller neoplasm size over time. Although the increase in the number of incidental NF-PNETs will continue in the future, we believe that the prognosis of PNET will improve. Therefore, it might be expected that this trend will continue and may impact the natural history of the disease.

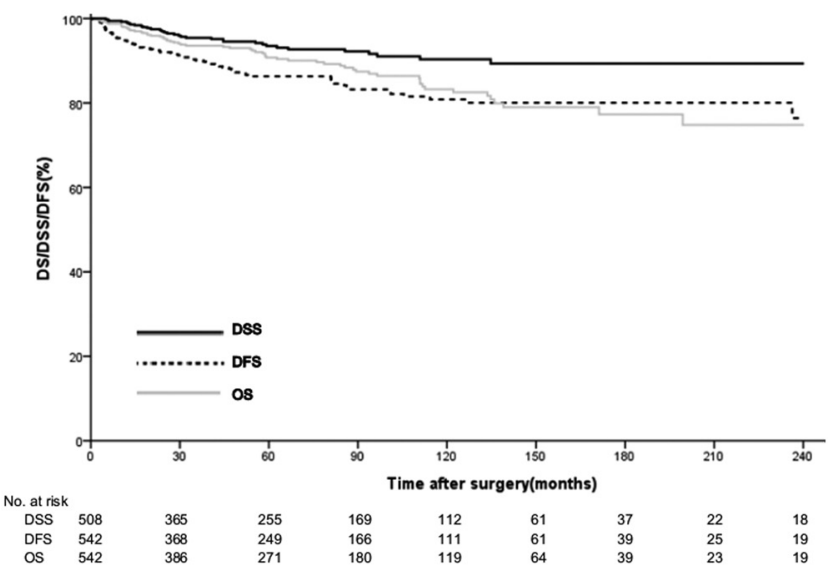

Figure 4

Overall survival (OS), disease-specific survival (DSS) and disease-free survival (DFS) in 542 patients with pancreatic neuroendocrine tumors (PNETs) after curative resection.

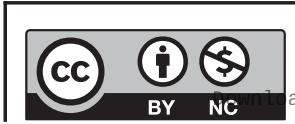

This work is licensed under a Creative Commons Attribution-NonCommercial 4.0 International License. ded from Bioscientifica.com at 04/26/2023 09:11:53AM via free access 
Table 5 Univariate and multivariate analysis of the predictors of DFS in the overall study population.

\begin{tabular}{l}
\hline \\
\hline Non-functioning tumors \\
G3 \\
T3/4 \\
N1 status \\
M1 status \\
LVI \\
PNI \\
Resection margin
\end{tabular}

\begin{tabular}{c}
\hline Univariate cox regr \\
\hline Hazard ratio \\
\hline $13.68(3.34-56.11)$ \\
$13.29(7.93-22.16)$ \\
$10.86(6.70-17.59)$ \\
$8.50(5.07-14.26)$ \\
$10.97(5.81-20.71)$ \\
$8.43(5.14-13.83)$ \\
$7.07(4.17-11.99)$ \\
$3.50(1.73-7.05)$ \\
\hline
\end{tabular}

\begin{tabular}{c} 
ression \\
\hline$<0.001$ \\
\hline$<0.001$ \\
$<0.001$ \\
$<0.001$ \\
$<0.001$ \\
$<0.001$ \\
$<0.001$ \\
$<0.001$
\end{tabular}

\begin{tabular}{ccc}
\hline \multicolumn{2}{c}{ Multivariate cox regression } \\
\hline Hazard ratio & & $P$ \\
\hline $4.51(2.32-8.78)$ & & $<0.001$ \\
$2.46(1.25-4.83)$ & & 0.009 \\
& \\
$3.62(1.57-8.34)$ & & 0.003 \\
$2.61(1.36-5.01)$ & 0.004 \\
$4.20(1.58-11.12)$ & 0.004 \\
\hline
\end{tabular}

Values in parentheses are $95 \% \mathrm{Cl}$.

LVI, lymphovascular invasion; PNI, perineural invasion.

Our data revealed excellent survival in patients with PNET, with the 5-year OS and DSS being 90.8 and $94.1 \%$, respectively, similar to 93 and $92.7 \%$, respectively, reported in another study $(13,14)$. Despite these good survival rates, especially in comparison to pancreatic ductal adenocarcinoma, prediction of the malignant potential of PNETs remains difficult. It is certainly important to evaluate DFS in PNET patients. Because of the relatively indolent nature of these tumors, survival can be prolonged even in the presence of recurrent tumors. Our study showed that 5- and 10-year DFS was 86.4 and $81.3 \%$, respectively. We analyzed patterns and predictors of recurrence in 542 surgically resected patients with PNET over a 27-year period. Although PNETS are generally considered to have a

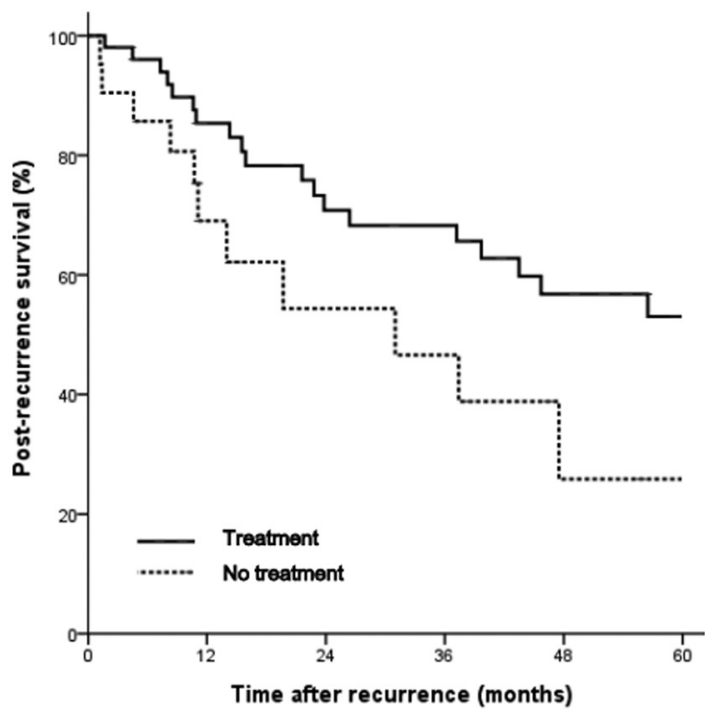

No. at risk

Treatment

No treatment$$
52
$$

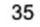

27

25

18

12

Figure 5

Survival time after recurrence for patients receiving treatment $(n=52)$ or with no treatment $(n=22) . P=0.06$ (log-rank test). low malignant potential, several patients experienced recurrence and had a poor prognosis. During the follow-up period, 74 (13.7\%) patients presented with postoperative recurrence, with a median time to recurrence of 23.2 (7.5-48.3) months. Four other studies, each with more than 100 patients, reported recurrence probabilities of $14.4-35 \%(13,15,16,17)$. Wei et al. reported that among 97 patients with intended curative resection of NF-PNETs, there were 14 (14.4\%) patients with recurrence involving the liver (67.9\%), remaining pancreas $(21.4 \%)$, lung $(7.1 \%)$, omentum $(7.1 \%)$, and peritoneum (7.1\%) (17). In this study, the organ that experienced recurrence most frequently was the liver $(54 / 74)$, followed by the liver plus other sites $(8 / 74)$, the retroperitoneum (4/74) and the peritoneum (3/74). There were also unexpected recurrence sites, such as the skin and the left supraclavicular lymph node.

On analysis, we found that significant clinicopathological predictors of DFS after curative resection of PNETs included a high tumor grade (G3), N1 status, M1 status, PNi, LVi and R1 status. There are a few studies in the literature citing the lymph node ratio and Ki67 index $>5 \%$ as predictors of PNET recurrence (18), in addition to other features such as multiple endocrine neoplasia type-1 syndrome and poorly differentiated neuroendocrine carcinoma (19), increased tumor size and increased Ki-67 staining (20), microscopic venous invasion and vascular invasion $(14,21)$ and $\mathrm{PNi}(22)$. The WHO grading system of proliferation of PNETs is based on the mitotic count and/or Ki67 index; this has been widely accepted as a good prognostic indicator for patients with PNET (23). These predictive factors should be considered as a part of the risk stratification to determine the therapeutic strategy for postoperative PNET.

Moreover, appropriate surveillance following resection of PNETs also remains an area of debate. The National Comprehensive Cancer Network guidelines

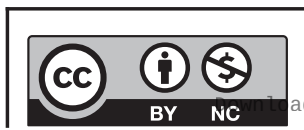

This work is licensed under a Creative Commons Attribution-NonCommercial 4.0 International License. ded from Bioscientifica.com at 04/26/2023 09:11:53AM via free access 
on PNETs recommend a complete history and physical examination and consideration of multiphasic CT or MRI once every 3-12 months following resection, and then every 6-12 months for up to 10 years (24). In our study, recurrence was highest $(81.7 \%)$ within 5 years; however, $3(4.2 \%)$ patients also showed hepatic recurrence after 10 years. Based on these findings, careful monitoring with repeat imaging studies may be necessary as per the current guidelines, particularly at 5 years post surgery. We also recommend that surveillance be used for patients at high risk of recurrence after 10 years. Patients with risk factors for recurrence should be monitored closely, with clinicians maintaining a high level of suspicion for indolent disease (which might recur late in the course of surveillance). Earlier identification of recurrent disease is important; this would allow for earlier medical and/or surgical intervention and, therefore, improve patient outcomes.

Lastly, our data showed that the median SAR was 22.6 (range 0.4-126.9) months. We provide multidisciplinary treatment for recurring PNETs in our centers. However, there was no significant $(P=0.06)$ difference in SAR according to the presence or absence of treatment. A few reports have shown that multi-disciplinary treatments, such as surgical resection, hormonal treatment and chemotherapy contribute to survival as part of the treatment strategy for recurring PNETs (25). Further studies to determine how to treat patients with recurrent PNETs are needed.

Our study is clearly limited by its reliance on data from a single institution and the retrospective nature of the data collection. Medical records were not complete for all patient groups, making it impossible to analyze all of the known predictors of disease recurrence and survival. Moreover, our patient population might not be representative of all centers caring for patients with PNETs in Korea. A follow-up study should entail a multi-center prospective trial, randomizing patients to standardized postoperative surveillance versus surveillance at an interval/frequency on the basis of clinically defined risk; this would allow evaluation of how such an optimized postoperative surveillance strategy affects patient outcomes.

In summary, with an increasing proportion of incidentally discovered NF-PNETs, the number of surgically resected PNETs has increased over time. Patients who undergo resection should be subject to long-term surveillance for risk factors including grade G3 PNETs, lymph node metastasis, LVi, $\mathrm{PNi}$ and a positive resection margin.
Declaration of interest

The authors declare that there is no conflict of interest that could be perceived as prejudicing the impartiality of the research reported.

\section{Funding}

This study was supported by a grant from the Korean Health Technology R\&D Project, Ministry of Health \& Welfare, Republic of Korea (No. HI14C2640).

\section{References}

1 Buchanan KD, Johnston CF, O'Hare MM, Ardill JE, Shaw C, Collins JS, Watson RG, Atkinson AB, Hadden DR \& Kennedy TL. Neuroendocrine tumors. A European view. American Journal of Medicine 198681 14-22. (https://doi.org/10.1016/00029343(86)90581-4)

2 Carriaga MT \& Henson DE. Liver, gallbladder, extrahepatic bile ducts, and pancreas. Cancer 199575 171-190. (https:// doi.org/10.1002/1097-0142(19950101)75:1+<171::aidcncr2820751306>3.0.co;2-2)

3 Fraenkel M, Kim MK, Faggiano A \& Valk GD. Epidemiology of gastroenteropancreatic neuroendocrine tumours. Best Practice and Research: Clinical Gastroenterology 201226 691-703. (https://doi. org/10.1016/j.bpg.2013.01.006)

4 Yao JC, Hassan M, Phan A, Dagohoy C, Leary C, Mares JE, Abdalla EK, Fleming JB, Vauthey JN, Rashid A, et al. One hundred years after 'carcinoid': epidemiology of and prognostic factors for neuroendocrine tumors in 35,825 cases in the united states. Journal of Clinical Oncology 200826 3063-3072. (https://doi.org/10.1200/ JCO.2007.15.4377)

5 Caldarella A, Crocetti E \& Paci E. Distribution, incidence, and prognosis in neuroendocrine tumors: a population based study from a cancer registry. Pathology Oncology Research 201117 759-763. (https://doi.org/10.1007/s12253-011-9382-y)

6 Haynes AB, Deshpande V, Ingkakul T, Vagefi PA, Szymonifka J, Thayer SP, Ferrone CR, Wargo JA, Warshaw AL \& Fernandez-del Castillo C. Implications of incidentally discovered, nonfunctioning pancreatic endocrine tumors: short-term and long-term patient outcomes. Archives of Surgery 2011146 534-538. (https://doi. org/10.1001/archsurg.2011.102)

7 Cheema A, Weber J \& Strosberg JR. Incidental detection of pancreatic neuroendocrine tumors: an analysis of incidence and outcomes. Annals of Surgical Oncology 201219 2932-2936. (https://doi. org/10.1245/s10434-012-2285-7)

8 Clavien PA, Barkun J, de Oliveira ML, Vauthey JN, Dindo D, Schulick RD, de Santibanes E, Pekolj J, Slankamenac K, Bassi C, et al. The clavien-dindo classification of surgical complications: fiveyear experience. Annals of Surgery 2009250 187-196. (https://doi. org/10.1097/SLA.0b013e3181b13ca2)

9 Fitzgerald TL, Smith AJ, Ryan M, Atri M, Wright FC, Law CH \& Hanna SS. Surgical treatment of incidentally identified pancreatic masses. Canadian Journal of Surgery $2003 \mathbf{4 6} 413-418$.

10 Fernandez-del Castillo C, Targarona J, Thayer SP, Rattner DW, Brugge WR \& Warshaw AL. Incidental pancreatic cysts: clinicopathologic characteristics and comparison with symptomatic patients. Archives of Surgery 2003138 427-423; discussion 433-424. (https://doi.org/10.1001/archsurg.138.4.427)

11 Vagefi PA, Razo O, Deshpande V, McGrath DJ, Lauwers GY, Thayer SP, Warshaw AL \& Fernandez-Del Castillo C. Evolving patterns in the detection and outcomes of pancreatic neuroendocrine neoplasms: the Massachusetts General Hospital experience from 1977 to 2005.

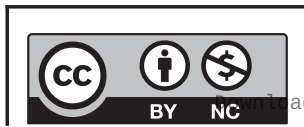

This work is licensed under a Creative Commons Attribution-NonCommercial 4.0 International License. ded from Bioscientifica.com at 04/26/2023 09:11:53AM 
Archives of Surgery 2007142 347-354. (https://doi.org/10.1001/ archsurg.142.4.347)

12 Bettini R, Partelli S, Boninsegna L, Capelli P, Crippa S, Pederzoli P, Scarpa A \& Falconi M. Tumor size correlates with malignancy in nonfunctioning pancreatic endocrine tumor. Surgery $2011 \mathbf{1 5 0}$ 75-82. (https://doi.org/10.1016/j.surg.2011.02.022)

$13 \mathrm{Xu}$ Han M \& Xuefeng DJ. Clinicopathological characteristics and prognosis related factors of resectable pnets. Pancreas 201443 526-531. (https://doi.org/10.1097/MPA.0000000000000065)

14 Landoni L, Marchegiani G, Pollini T, Cingarlini S, D'Onofrio M, Capelli P, De Robertis R, Davi MV, Amodio A, Impellizzeri H, et al. The evolution of surgical strategies for pancreatic neuroendocrine tumors (pan-nens): time-trend and outcome analysis from 587 consecutive resections at a high-volume institution. Annals of Surgery 2019269 725-732. (https://doi.org/10.1097/ SLA.0000000000002594)

15 Fischer L, Bergmann F, Schimmack S, Hinz U, Priess S, MullerStich BP, Werner J, Hackert T \& Buchler MW. Outcome of surgery for pancreatic neuroendocrine neoplasms. British Journal of Surgery 2014 101 1405-1412. (https://doi.org/10.1002/bjs.9603)

16 Wong J, Fulp WJ, Strosberg JR, Kvols LK, Centeno BA \& Hodul PJ. Predictors of lymph node metastases and impact on survival in resected pancreatic neuroendocrine tumors: a single-center experience. American Journal of Surgery 2014208 775-780. (https:// doi.org/10.1016/j.amjsurg.2014.04.003)

17 Wei IH, Harmon CM, Arcerito M, Cheng DF, Minter RM \& Simeone DM. Tumor-associated macrophages are a useful biomarker to predict recurrence after surgical resection of nonfunctional pancreatic neuroendocrine tumors. Annals of Surgery 2014260 1088-1094. (https://doi.org/10.1097/SLA.0000000000000262)

18 Boninsegna L, Panzuto F, Partelli S, Capelli P, Delle Fave G, Bettini R, Pederzoli P, Scarpa A \& Falconi M. Malignant pancreatic neuroendocrine tumour: lymph node ratio and Ki67 are predictors of recurrence after curative resections. European Journal of Cancer 2012 48 1608-1615. (https://doi.org/10.1016/j.ejca.2011.10.030)
19 Casadei R, Ricci C, Pezzilli R, Campana D, Tomassetti P, Calculli L, Santini D, D'Ambra M \& Minni F. Are there prognostic factors related to recurrence in pancreatic endocrine tumors? Pancreatology 201010 33-38. (https://doi.org/10.1159/000217604)

20 Hamilton NA, Liu TC, Cavatiao A, Mawad K, Chen L, Strasberg SS, Linehan DC, Cao D \& Hawkins WG. Ki-67 predicts disease recurrence and poor prognosis in pancreatic neuroendocrine neoplasms. Surgery 2012152 107-113. (https://doi.org/10.1016/j.surg.2012.02.011)

21 Nanno Y, Toyama H, Otani K, Asari S, Goto T, Terai S, Ajiki T, Zen Y, Fukumoto T \& Ku Y. Microscopic venous invasion in patients with pancreatic neuroendocrine tumor as a potential predictor of postoperative recurrence. Pancreatology 201616 882-887. (https:// doi.org/10.1016/j.pan.2016.06.008)

22 Genc CG, Jilesen AP, Partelli S, Falconi M, Muffatti F, van Kemenade FJ, van Eeden S, Verheij J, van Dieren S, van Eijck CHJ, et al. A new scoring system to predict recurrent disease in grade 1 and 2 nonfunctional pancreatic neuroendocrine tumors. Annals of Surgery 2018267 1148-1154. (https://doi.org/10.1097/ SLA.0000000000002123)

23 Scarpa A, Mantovani W, Capelli P, Beghelli S, Boninsegna L, Bettini R, Panzuto F, Pederzoli P, delle Fave G \& Falconi M. Pancreatic endocrine tumors: improved tnm staging and histopathological grading permit a clinically efficient prognostic stratification of patients. Modern Pathology 201023 824-833. (https://doi.org/10.1038/modpathol.2010.58)

24 Kulke MH, Shah MH, Benson AB, Bergsland E, Berlin JD, Blaszkowsky LS, Emerson L, Engstrom PF, Fanta P, Giordano T, et al. Neuroendocrine Tumors, Version 1.2015. Journal of the National Comprehensive Cancer Network 201513 78-108. (https://doi. org/10.6004/jnccn.2015.0011)

25 Zhang XF, Beal EW, Chakedis J, Lv Y, Bagante F, Aldrighetti L, Poultsides GA, Bauer TW, Fields RC, Maithel SK, et al. Early recurrence of neuroendocrine liver metastasis after curative hepatectomy: risk factors, prognosis, and treatment. Journal of Gastrointestinal Surgery 201721 1821-1830. (https://doi.org/10.1007/ s11605-017-3490-2)

Received in final form 11 June 2019

Accepted 27 June 2019

Accepted Preprint published online 28 June 2019 https://ec.bioscientifica.com

https://doi.org/10.1530/EC-19-0282
(C) 2019 The authors Published by Bioscientifica Ltd
This work is licensed under a Creative Commons Attribution-NonCommercial 4.0 International License. ded from Bioscientifica.com at 04/26/2023 09:11:53AM 